\title{
Reduction in seizure burden in a child with a A350V IQSEC2 mutation using a Jacuzzi
}

\author{
Andrew Levy ${ }^{1}$, Nina Levy ${ }^{1}$, Eli Heyman ${ }^{2}$, Mitchell Schertz $^{1}$, and Jacob Genizi ${ }^{1}$ \\ ${ }^{1}$ Technion Israel Institute of Technology \\ ${ }^{2}$ Assaf Harofeh Medical Center
}

June 18, 2021

\begin{abstract}
A child with a A350V IQSEC2 missense mutation resulting in drug resistant epilepsy stops having seizures when he has a fever. We demonstrate that raising the body temperature of the child using a commercial Jacuzzi dramatically reduces his seizures and appears to improve his social behavioral interactions.
\end{abstract}

\section{Reduction in seizure burden in a child with a A350V IQSEC2 mutation using a Jacuzzi}

Andrew P Levy ${ }^{1 *}$, Nina S Levy ${ }^{1}$, Eli Heyman ${ }^{2}$, Mitchell Schertz $^{3}$, Jacob Genizi ${ }^{4}$

${ }^{1}$ Department of Biochemistry, Technion Faculty of Medicine, Technion Israel Institute of Technology, Haifa Israel $;{ }^{2}$ Department of Pediatric Neurology, Assaf Harofeh Medical Center and Tel Aviv University, Tel Aviv, Israel ${ }^{3}$ Child Development and Pediatric Neurology Service, Mehudet North and Technion Israel Institute of Technology, Haifa, Israel; ${ }^{4}$ Pediatric Neurology Unit, Bnai Zion Medical Center and Technion Israel Institute of Technology, Haifa, Israel;

*Correspondence: Andrew P Levy, Technion Faculty of Medicine, Technion Israeli Institute of Technology, Haifa, Israel. Email: alevy@technion.ac.il;Tel: 972-528664296

Word count: 2046

Figures: 2

\section{Key Clinical Message}

A child with a A350V IQSEC2 missense mutation resulting in drug resistant epilepsy stops having seizures when he has a fever. We demonstrate that raising the body temperature of the child using a commercial Jacuzzi dramatically reduces his seizures and appears to improve his social behavioral interactions.

Keywords : IQSEC2, heat shock, thermal therapy, epilepsy, child development

\section{Introduction}

\section{A350V IQSEC2 mutation and features of its associated seizures prompting this case report}

Mutations in the X-linked human IQSEC2 gene (NM_001111125) (MIM 300522) are associated with epilepsy as well as severe disturbances in cognitive and social behavioral functioning [1-4]. Seizures in male children with IQSEC2 mutations are drug resistant in over $80 \%$ of cases [3,4]. Over the past several years we have studied the pathophysiology and clinical course of one specific human IQSEC2 missense mutation (A350V defined as a valine for alanine substitution at amino acid residue number 350 of the IQSEC2 protein) $[5,6]$. 
Over 20 different seizure medications and a ketogenic diet have failed to reduce seizures in the male child E.H. hemizygous for the A350V IQSEC2 mutation. The failure of conventional medical treatment to suppress the child's seizures has prompted our group to investigate alternative approaches. A remarkable finding in this child repeatedly observed by his parents beginning at one year of age until the present (age 8) is that when he has a fever of greater than $38^{\circ} \mathrm{C}$ lasting more than 1 day his seizures abruptly stop and then he has a seizure free window of up to 3 weeks after the fever has resolved. In this case report we set out to determine if the purposeful induction of a rise in body core temperature could have the same benefit as fever on reducing seizures.

\section{Case report}

We have previously reported on the developmental progression of EH thru age 5 including the onset of seizures at age 8 months and the refractory nature of these seizures to all treatments [5]. Seizure activity has been repeatedly documented using video EEG with the most recent EEG prior to this study (12/2019) showing nearly continuous severe epileptiform activity which was well correlated with frequent short head drops as well as generalized tonic clonic (GTC) seizures and drop attacks. Prior to beginning this current investigation, the frequency of different seizure types was very variable from day to day but it was not uncommon for the child to have over 20 seizures a day and due to post-ictal effects to be lethargic and non-interactive a substantial portion of the day.

We considered several possibilities to try to raise the child's body core temperature to see if we could recapitulate the apparent protective effect of fever on seizures as described above. Previous studies have established that 15-20 minute exposures to water baths at $40^{\circ} \mathrm{C}$ can safely raise the body core temperature to up to $39^{\circ} \mathrm{C}$ without risk of hyperthermia [7-9]. We chose for this purpose a temperature controlled water bath (Coleman 13804-BW SaluSpa) which is sold commercially as a Jacuzzi and operated the Jacuzzi at a setting of $40^{\circ} \mathrm{C}$. The child was encouraged to sit in the Jacuzzi for treatments of up to 20 minutes and was monitored closely by an adult caregiver. More often the child would jump around and play during these treatments.

Beginning in October 2020, two months prior to initiating the Jacuzzi treatments the child's seizures were carefully documented for two to three hours a day by a single caretaker and recorded as being either small (head drops) or large (tonic-clonic followed by post-ictal drowsiness lasting at least 15 minutes). Once daily outdoor Jacuzzi treatments without any control of the air temperature of the region surrounding the Jacuzzi (temperature outside the Jacuzzi ranging from $10-15^{\circ} \mathrm{C}$ ) were initiated in December 2020 with no apparent benefit on the number of head drops or GTC seizures. Beginning in February 2021 twice daily Jacuzzi treatments were initiated in conjunction with the housing of the Jacuzzi inside a tent and the installation of a heating system in the tent allowing the air temperature in the tent to be controlled at $30^{\circ} \mathrm{C}$ during the Jacuzzi treatments. Figure 1 demonstrates the number of days per month the child was observed to have at least two observed head drops or one GTC seizure over the four-month period prior to beginning the twice daily Jacuzzi treatments and during the one-month period when he received twice daily treatments (average of 7-11 days per month prior to twice daily treatments compared to one day during the month of twice daily treatments, $\mathrm{p}=0.003)$.

During the one-month period in which the child received twice daily Jacuzzi treatments the child was more alert and interactive with his caregivers and teachers. His sleep was also markedly improved. Although the child is averbal, during this period the child demonstrated increased vocalizations.

After one month of twice daily Jacuzzi treatments an EEG was performed (March 2021) and demonstrated a marked improvement in the amount of epileptiform activity. Representative EEGs from the period before and after the twice daily Jacuzzi treatments are shown in Figure 2. Compared to his most recent EEG done prior to the Jacuzzi treatments (Dec 2019) there was a marked reduction in interictal discharges (A1 prior and A2 after treatment) (mean of 6 interictal discharges per minute compared to one interictal discharge per minute). Moreover, background brain wave activity of the EEG after treatment was markedly improved (B1 prior and B2 after treatment) with normal sleep patterns after twice daily Jacuzzi treatments and a 
reduction in delta wave activity seen prior to treatment.

\section{Discussion and Outlook}

In this case report we have observed objective benefit of frequent treatments in a $40^{\circ} \mathrm{C}$ Jacuzzi on seizure burden in a child with intractable epilepsy. This may be mediated by a similar biological process as seen in the child when he has had a fever in the past and had stopped having seizures. We are currently investigating the mechanism for this apparent relationship between body core temperature and seizures with the A350V IQSEC2 mutation in preclinical models using patient derived stem cells and in a CRISPR generated mouse model of the A350V IQSEC2 mutation. We have proposed that the protection by elevated temperature may be mediated by the induction of specific heat shock proteins which can act as molecular chaperones aiding in the refolding of denatured or misfolded (as from a missense mutation) proteins (10-16). In general, such chaperones recognize newly exposed hydrophobic domains of proteins with the alanine to valine amino acid substitution in A350V possibly providing such an epitope. It is not clear whether children with different IQSEC2 mutations might be helped with this treatment. We hope that the reduction in seizure burden which this treatment appears to provide to a child with the A350V IQSEC2 mutation may permit for at least a partial improvement in his neurodevelopmental progression.

Acknowledgments. We would like to thank David Lieberman for providing the Jacuzzi as a gift. No sources of funding are declared for this study.

Approval and consent. The parents of EH provided informed consent for his Jacuzzi treatments and to use the data from his seizures in this case report.

Authorship. APL: designed the study, wrote the case report; NSL: helped with writing the case report; EH: obtained EEG, helped to review manuscript; MS: helped to write the case report; JG: helped to write the case report, obtained EEG and performed comparative analysis of EEG pre and post treatment.

Conflict of interest. none

\section{Figures}

Figure 1. Seizure burden before and after twice daily Jacuzzi treatments. Histogram showing the number of days per 30-day interval in which EH was observed to have at least two head drops or one GTC seizure. Documentation of seizures was initiated October 10, 2019. Once daily Jacuzzi treatments without control of air temperature were initiated in the beginning of December 2020 and twice daily treatments with maintenance of air temperature at $30^{\circ} \mathrm{C}$ were initiated on February 10, 2021. Time periods in histogram are: period A-October 10, 2019-November 10, 2019; period B-November 11, 2019- December 10, 2019; period C-December 11, 2019-January 10, 2020; period D- January 11, 2021-Febuary 10, 2021; period E-February 11, 2021 to March 10, 2021. (***, $\mathrm{p}=0.003$ for seizures prior and after twice daily Jacuzzi treatments).

Figure 2. Differences in epileptiform and baseline brain wave activity seen on EEG before (Dec 2019) and after (March 2021) twice daily Jacuzzi treatments. There was a marked reduction in interictal discharges seen after initiating Jacuzzi treatments $(6$ per minute before treatment as shown in A1, compared to 1 per minute after treatment as shown in A2). The distribution of brain wave activity showed prominent delta wave activity prior to treatment (B1) compared to normal sleep patterns after treatment (B2).

\section{A.1. Interictal EEG discharges before Jacuzzi treatment}

\section{A.2. Interictal EEG discharges after Jacuzzi treatment}

\section{B.1. Background EEG activity before Jacuzzi treatment showing delta waves}

B.2. Background EEG activity after Jacuzzi treatment showing normal sleep patterns

\section{References}


1. Shoubridge, C., P.S. Tarpey, F. Abidi, S.L. Ramsden, S. Rujirabenjerd, J.A. Murphy, et al. 2010. Mutations in the guanine nucleotide exchange factor gene IQSEC2 cause nonsyndromic intellectual disability. Nat. Genet. 42:486-488.

2. Zerem, A., K. Haginoya, D. Lev, L. Blumkin, S. Kivity, I. Linder, et al. 2016. The molecular and phenotypic spectrum of IQSEC2-related epilepsy. Epilepsia 57:1858-1869.

3. Shoubridge C., R.J. Harvey, and T. Dudding-Byth. 2019. IQSEC2 mutation update and review of the female-specific phenotype spectrum including intellectual disability and epilepsy. Human Mutation 40: $5-24$.

4. Mignot C, and C. Depienne. 2019. IQSEC2 related encephalopathy in males and females: a comparative study including 37 novel patients. Genetics in Medicine 21: 837-849

5. Zipper, R., S.D, Baine, J. Genizi, H. Maoz, N.S. Levy, and A.P. Levy. 2017. Developmental progression of intellectual disability, autism and epilepsy in a child with an IQSEC2 gene mutation. Clinical Case Reports 5:1639-1643.

6. Rogers E.J., R. Jada, K. Schagenheim-Rozales, M. Sah, M. Cortes, M. Florence, et al. 2019. An IQSEC2 mutation associated with intellectual disability and autism results in decreased surface AMPA receptors. Front Mol Neuroscience 12:43.

7. Faulkner S.H., S. Jackson, G. Fatania, and C.A. Leicht. 2017. The effect of passive heating on heat shock protein 70 and interleukin-6: A possible treatment tool for metabolic diseases? Temperature 4:292-304.

8. Allison T.G., and W.E. Reger. 1998. Comparison of responses of men to immersion in circulating water at 40.0 and 41.5 degrees C. Aviat. Space Environ. Med. 69:845-850.

9. Miwa C., T. Matsukawa, S. Iwase, Y. Sugiyama, T. Mano, J. Sugenoya, et al. 1994. Human cardiovascular responses to a 60-min bath at 40 degrees C. Environ. Med. 38:77-80.

10. Hunt A.P., G.M. Minett, O.R. Gibson, G.K. Kerr, and I.B. Stewart. 2020. Could heat therapy be an effective treatment for Alzheimer's and Parkinson's disease? A narrative review. Front. Physiology 10:1556.

11. Paul S., and S. Mahanta. 2014. Association of heat shock proteins in various neurodegenerative disorders: is it a master key to open the therapeutic door? Mol. Cell Biochem. 386:45-61.

12. Hasday J.D., and I.S. Singh. 2000. Fever and heat shock response: distinct, partially overlapping processes. Cell Stress and Chap. 5:471-480.

13. Duveau V., S. Arthand, H. Serre, A. Rougier, and G.L.G. La Salle. 2005. Transient hyperthermia protects against subsequent seizures and epilepsy induced cell damage in the rat. Neurobio. Dis. 19:142149

14. Karunanithi S., and I.R. Brown. 2015. Heat shock response and homeostatic plasticity. Front Cell. Neuro. 9:68.

15. Luengo T.M., M.P. Mayer, and S.G.D. Rudiger. 2019. The HSP 70-Hsp 90 chaperone cascade in protein folding. Trends in Cell. Biol. 29:164

16. Karras G.I., S. Yi, N. Sahni, M. Fischer, J. Xie, M. Vidal, et al. 2017. HSP 90 shapes the consequences of human genetic variation. Cell 168:856-866. 

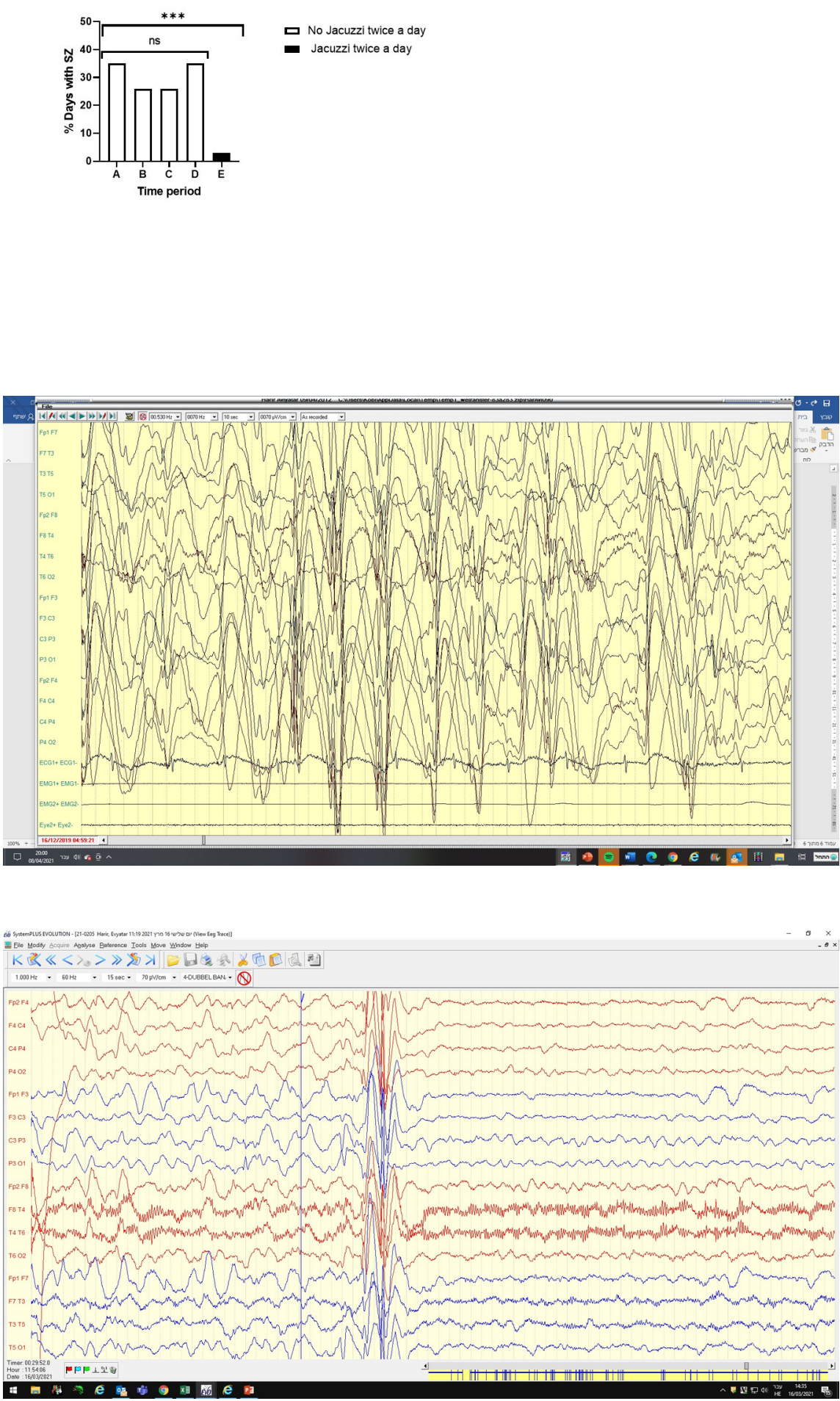

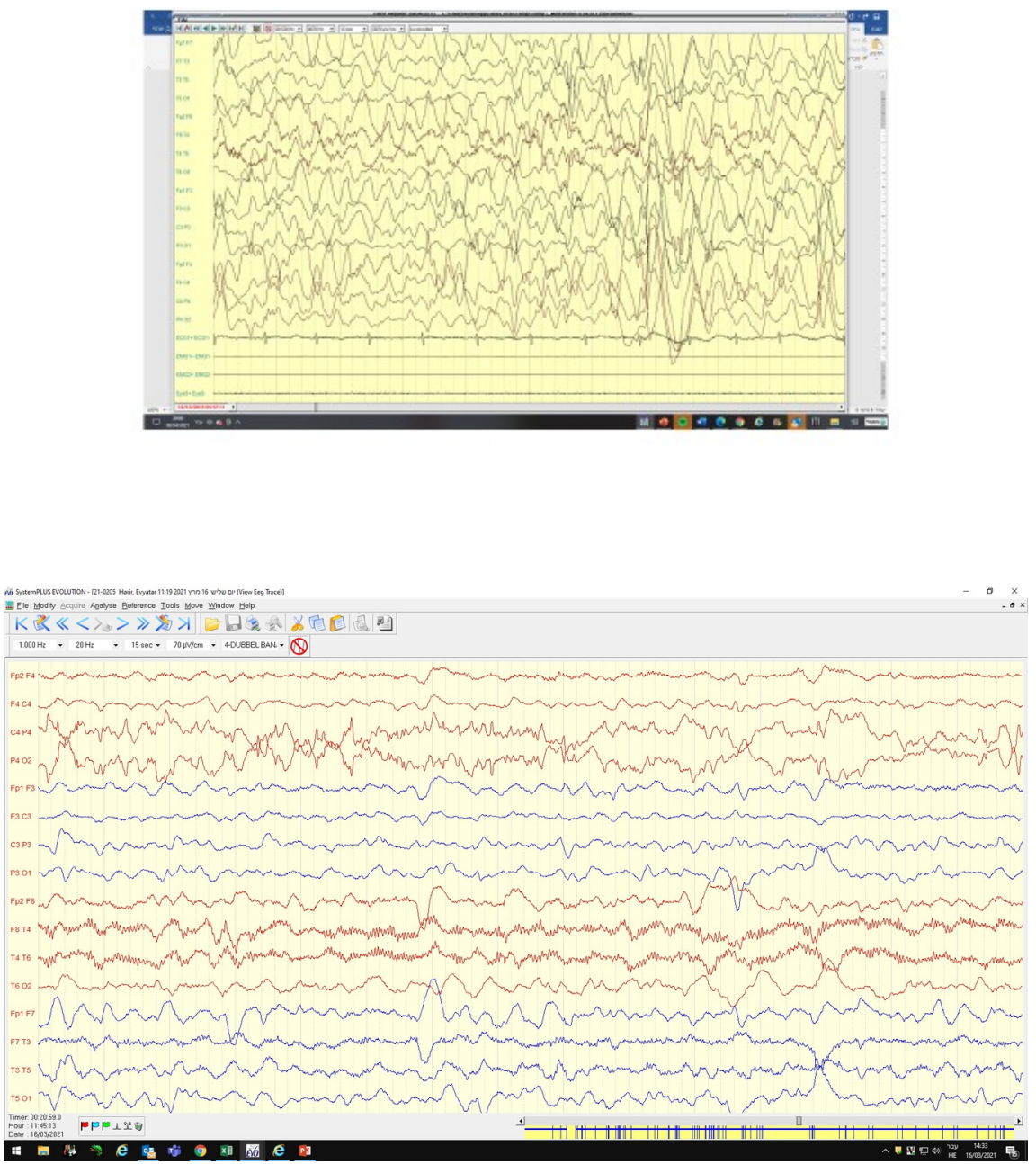